\title{
Ability of ICU Health-Care Professionals to Identify Patient- Ventilator Asynchrony Using Waveform Analysis
}

\author{
Ivan I Ramirez PT, Daniel H Arellano PT CRT MSc, Rodrigo S Adasme PT CRT, \\ Jose M Landeros PT CRT, Francisco A Salinas PT, Alvaro G Vargas PT CRT, \\ Francisco J Vasquez PT CRT, Ignacio A Lobos PT, Magdalena L Oyarzun PT CRT, and \\ Ruben D Restrepo MD RRT FAARC
}

\begin{abstract}
BACKGROUND: Waveform analysis by visual inspection can be a reliable, noninvasive, and useful tool for detecting patient-ventilator asynchrony. However, it is a skill that requires a properly trained professional. METHODS: This observational study was conducted in 17 urban ICUs. Health-care professionals (HCPs) working in these ICUs were asked to recognize different types of asynchrony shown in 3 evaluation videos. The health-care professionals were categorized according to years of experience, prior training in mechanical ventilation, profession, and number of asynchronies identified correctly. RESULTS: A total of 366 HCPs were evaluated. Statistically significant differences were found when HCPs with and without prior training in mechanical ventilation (trained vs non-trained HCPs) were compared according to the number of asynchronies detected correctly (of the HCPs who identified 3 asynchronies, 63 [81\%] trained vs 15 [19\%] non-trained, $P<.001 ; 2$ asynchronies, 72 [65\%] trained vs 39 [35\%] non-trained, $P=.034 ; 1$ asynchrony, 55 [47\%] trained vs 61 [53\%] non-trained, $P=.02 ; 0$ asynchronies, 17 [28\%] trained vs 44 [72\%] non-trained, $P<.001)$. HCPs who had prior training in mechanical ventilation also increased, nearly 4-fold, their odds of identifying $\geq 2$ asynchronies correctly (odds ratio $3.67,95 \%$ CI 1.93-6.96, $P<.001)$. However, neither years of experience nor profession were associated with the ability of HCPs to identify asynchrony. CONCLUSIONS: HCPs who have specific training in mechanical ventilation increase their ability to identify asynchrony using waveform analysis. Neither experience nor profession proved to be a relevant factor to identify asynchrony correctly using waveform analysis. Key words: critical care; mechanical ventilation; intensive care unit; patient-ventilator asynchrony; ventilator graphics; waveforms. [Respir Care 2017;62(2):144-149. () 2017 Daedalus Enterprises]
\end{abstract}

\section{Introduction}

Patient-ventilator interaction is described as "an expression of the function of 2 controllers (the ventilator con-

\footnotetext{
Mr Ramirez and Mr Arellano are affiliated with the Division of Critical Care Medicine, Hospital Clinico Universidad de Chile, Santiago, Chile. Mr Adasme is affiliated with the Division of Critical Care Medicine, Hospital Clinico Universidad Catolica, Santiago, Chile and Epidemiology Master Degree, Faculty of Medicine, Universidad de Los Andes. Mr Landeros is affiliated with the Division of Critical Care Medicine Hospital Roberto del Rio, Santiago, Chile. Mr Salinas is affiliated with the Division of Critical Care Medicine, Instituto Nacional del Torax, Santiago, Chile. Mr Vargas is affiliated with the Division of Critical Care Medicine, Hospital Higueras de Talcahuano, Chile. Mr Vasquez is affiliated with the Division of Critical Care Medicine, Hospital de Talca,
}

trolled by the physician and the patient's own respiratory muscle pump) which should be in harmony if the result is to be appropriate for the patient." ${ }^{1}$ Therefore, patientventilator asynchrony can be described as "any condition where the patient-ventilator interaction is not optimal."2

\footnotetext{
Talca, Chile. Mr Lobos is affiliated with the Division of Critical Care Medicine, Hospital Clinico de la Florida, Santiago, Chile. Ms Oyarzun is affiliated with the Division of Critical Care Medicine, Clinica Bicentenario, Santiago Chile. Dr Restrepo is affiliated with the Department of Respiratory Care, University of Texas Health Sciences Center at San Antonio, San Antonio, Texas.
}

The authors have disclosed no conflicts of interest 
Several studies have described the complications associated with asynchrony. Some of them are increased work of breathing, ${ }^{3}$ delayed and prolonged weaning, ${ }^{4}$ gas exchange alterations, ${ }^{5}$ and longer mechanical ventilation duration and ICU stay. ${ }^{6,7}$ This shows that asynchrony interferes with the objective of mechanical ventilation, which should be (1) decreasing work of breathing, (2) maintaining adequate gas exchange, and (3) unloading the respiratory muscles. ${ }^{2,8,9}$ So it is important that health-care professionals (HCPs) are properly trained to identify asynchrony.

\section{See the Related Editorial on Page 248}

There are several tools to assess asynchrony. ${ }^{1,4,7,10-13}$ Measurement of diaphragmatic electrical activity by electromyography and esophageal pressure measurement have been considered the methods of choice for detecting asynchrony. ${ }^{2,4,7}$ However, they are invasive and expensive; therefore, their availability during daily clinical practice is limited. 3,5 On the other hand, evaluation using waveform analysis is not only a noninvasive and reliable alternative method, but it also has shown good correlation with invasive methods. ${ }^{7}$

There are no reports in the literature regarding HCP knowledge of ventilator graphics according to their prior training in mechanical ventilation. Also, there are no reports indicating whether training in mechanical ventilation, experience, or profession are associated with the ability of HCPs to identify asynchrony correctly using waveform analysis. The aim of our study was to assess the ability of ICU HCPs to identify different types of asynchronies according to their years of experience, profession, and prior training in mechanical ventilation using waveform analysis.

\section{Methods}

Ethical committee approval was not required by the institutions, because no assessment/procedure was performed in patients. Every HCP assessed in the study voluntarily agreed to participate.

This was a descriptive/observational study in which an evaluation tool designed and validated by the investigators, which consisted of 3 videos of asynchronies and a data gathering/evaluation sheet, was sent to 25 hospitals and distributed among different ICU HCPs, including physiotherapists, physicians, and nurses. An evaluator, who remained blinded to study participants, was selected at

Correspondence: Daniel H Arellano PT CRT MSc, Intensive Care Unit, Hospital Clinico Universidad de Chile, Santos Dumont 999, Independencia, Santiago, Chile. E-mail: darellano@vtr.net.

DOI: $10.4187 /$ respcare .04750

\section{QUICK LOOK}

\section{Current knowledge}

Waveform analysis from mechanical ventilation is a noninvasive method to identify patient-ventilator asynchronies. The ability of health-care professionals working in the ICU is influenced by experience according to some reports. However, studies have not reported the influence of training in mechanical ventilation, years of experience, and profession.

\section{What this paper contributes to our knowledge}

Health-care professionals who have specific training in mechanical ventilation increase their ability to identify patient-ventilator asynchrony correctly using waveform analysis. However, the profession of each participant and the years of experience working in the ICU did not influence the ability to identify asynchronies.

each institution to conduct the assessments and asked to adhere to an answer key provided by the investigators.

Before evaluation, each participant was asked to state in the registration/evaluation paper their profession and years of experience and to specify whether they had prior training in mechanical ventilation. Professionals were further categorized according to years of experience working and managing mechanical ventilation at the ICU (experience and limited experience) and prior training in mechanical ventilation (trained and non-trained). We defined experienced HCPs as HCPs who had been working and managing mechanical ventilation at their ICUs for $\geq 5 \mathrm{y}$. Limited-experience HCPs were those who had been working and managing mechanical ventilation at their ICUs for $<5$ y. Trained HCPs were defined as those who had successfully completed at least one course focus (entirely) in mechanical ventilation from a formal educational institution (university or college) and with a specific curriculum that included modes of mechanical ventilation, patientventilator synchrony, and waveform analysis among the topics.

Three videos of asynchrony (double-triggering, autotriggering, and ineffective effort) were presented to each HCP (in the same order) (Fig. 1). After each video was shown, the HCPs were asked to select the best answer from a multiple-choice question in the evaluation sheet. Each video had 5 different choices. For the first video (double-triggering), the options presented were: (a) autotriggering, (b) delayed cycling, (c) double-triggering, (d) flow asynchrony, and (e) there is not asynchrony. For the second video (autotriggering), the choices were: (a) double-triggering, (b) autotriggering, (c) delayed cycling, (d) premature cycling, and (e) there is not asynchrony. For the 

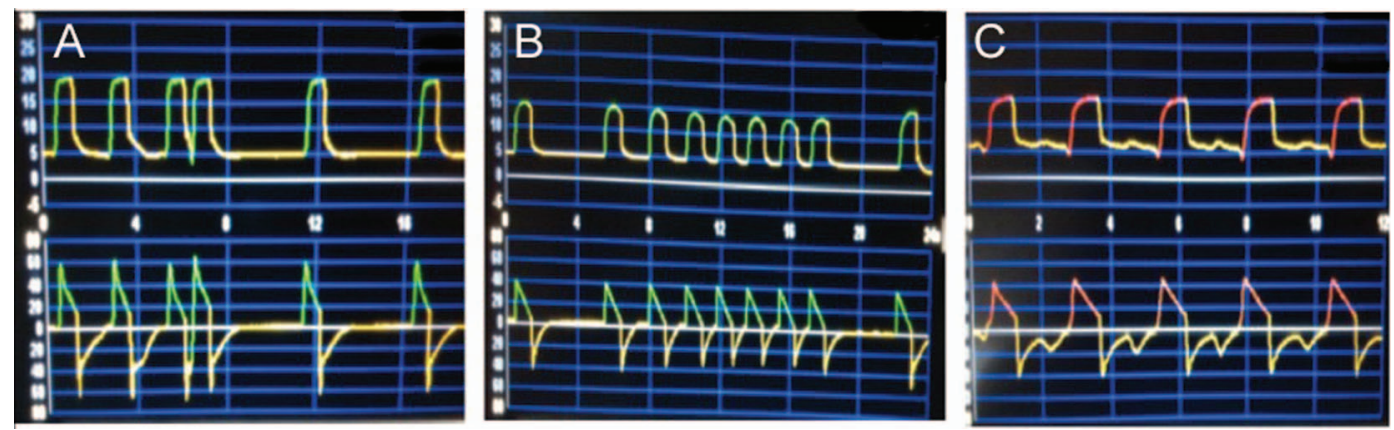

Fig. 1. Extract from the videos showed on the evaluation to each professional. Each video presents the pressure/time and flow/time waveforms. Shown are double-triggering $(A)$, autotriggering $(B)$, and ineffective effort (C).

third video (ineffective effort), the choices were: (a) autotriggering, (b) ineffective effort, (c) flow asynchrony, (d) double-triggering, and (e) there is not asynchrony.

Each video was recorded from a Puritan Bennett 840 ventilator (Covidien, Carlsbad, California) and showed only 2 scalars: pressure versus time and flow versus time waveforms. The asynchronies were simulated by using a test lung connected to the mechanical ventilator. Double-triggering was defined as " 2 consecutive inspirations occurring within an interval of less than half of the mean inspiratory time." 14 To simulate double-triggering, a negative pressure within the test lung was created by performing a decompression right after the inspiratory time ended. Autotriggering was defined as "a delivery of a breath that is neither scheduled (based on the set respiratory frequency) nor initiated by the patient." ${ }^{14}$ To simulate autotriggering, a minimal leak was created in the system (by partially disconnecting the test lung from the mechanical ventilator and programing a high sensitivity) to drop airway pressure only enough to trigger a breath. Ineffective effort was defined as "patient efforts that are not sensed by the ventilator." 14 To simulate ineffective effort, a negative pressure within the test lung was created by performing a decompression during the expiratory time of the breath.

To properly understand the asynchronies shown in the videos, we also defined (a) the trigger variable as "the variable that start[s] inspiration," 15 (b) the cycle variable as "the variable (usually pressure, volume, flow, or time) that is measured and used to end inspiration (and begins expiratory flow),"15 (c) assisted breath as "a breath during which all or part of inspiratory (or expiratory) flow is generated by the ventilator doing work on the patient," 15 (d) breath as "a positive change in airway flow (inspiration) paired with a negative change in airway flow (expiration), associated with ventilation of the lungs," 15 and (e) sensitivity as "a threshold value for the trigger variable that, when met, starts inspiration." 15

To validate the assessment tools, the videos were shown to 10 different HCPs, each of them expert in the field of critical care and responsible for mechanical ventilation management at their hospitals. For the 3 types of asynchrony, the inter-observer agreement was $100 \%$.

\section{Statistical Analysis}

STATA 13.0 (StataCorp, College Station, Texas) was used for data analysis. Medians and interquartile range were used to express continuous data (years of experience). To describe the variables, we used absolutes and relatives frequencies. The chi-square and Fisher exact tests were used to compare qualitative variables according to the number of asynchronies identified correctly. To analyze qualitative variables (experience and prior training in mechanical ventilation), HCPs were categorized as experienced, limited experienced, trained, and non-trained. To determine the association between prior training in mechanical ventilation, years of experience, and profession and the ability of HCPs to identify $\geq 2$ asynchronies correctly, we used a binary multiple logistic regression model (this model was adjusted by experience and profession). To perform this analysis, we considered whether the participants detected $\geq 2$ asynchronies correctly as our outcome variable and the group of nurses as our reference category for profession (reference cell). A $P$ value of $<.05$ was considered statistically significant for all analysis.

\section{Results}

Seventeen centers (68\% response rate) participated. A total of $366 \mathrm{HCPs}$, including 120 physiotherapists (32.8\%), 88 physicians (24.0\%), and 158 nurses (43.2\%), completed the evaluation. The general characteristics of HCPs according to profession are summarized in Tables 1 and 2 .

Only 78 HCPs $(21.3 \%)$ recognized the 3 types of asynchrony correctly, whereas $111(30.3 \%)$ detected 2 types correctly, and $116(31.7 \%)$ detected 1 type correctly. Sixty-one participants $(16.7 \%)$ did not identify any asynchrony. Double-triggering was identified by $213(58.2 \%)$ of the HCPs, autotriggering by 181 (49.4\%), and ineffective effort by $178(48.6 \%)$. 
Table 1. Characteristics of Health-Care Professionals

\begin{tabular}{lc}
\hline \hline \multicolumn{1}{c}{ Characteristics } & Values $(N=366)$ \\
\hline Years of experience, median (IQR) & $3(2-6)$ \\
Trained, $n(\%)$ & $207(56.6)$ \\
Non-trained, $n(\%)$ & $159(43.4)$ \\
Experienced, $n(\%)$ & $144(39.3)$ \\
Limited-experienced, $n(\%)$ & $222(60.7)$ \\
Experienced, trained, $n(\%)$ & $90(24.6)$ \\
Experienced, non-trained, $n(\%)$ & $54(14.8)$ \\
Limited-experienced, trained, $n(\%)$ & $117(31.9)$ \\
Limited-experienced, non-trained, $n(\%)$ & $105(28.6)$ \\
& \\
IQR $=$ interquartile range &
\end{tabular}

Table 2. Characteristics of Health-Care Professionals According to Profession

\begin{tabular}{lccc}
\hline \hline \multicolumn{1}{c}{ Characteristics } & $\begin{array}{c}\text { Physiotherapists } \\
(n=120)\end{array}$ & $\begin{array}{c}\text { Physicians } \\
(n=88)\end{array}$ & $\begin{array}{c}\text { Nurses } \\
(n=158)\end{array}$ \\
\hline Years of experience, median (IQR) & $2(1-5)$ & $4(2-9)$ & $4(2-7)$ \\
Trained, $n(\%)$ & $92(76.7)$ & $53(60.2)$ & $62(39.2)$ \\
Non-trained, $n(\%)$ & $28(23.3)$ & $35(39.8)$ & $96(60.8)$ \\
Experienced, $n(\%)$ & $32(26.7)$ & $43(48.9)$ & $69(43.7)$ \\
Limited-experienced, $n(\%)$ & $88(73.3)$ & $45(51.1)$ & $89(56.3)$ \\
Experienced, trained $n(\%)$ & $28(23.3)$ & $29(32.9)$ & $33(20.8)$ \\
Experience, non-trained, $n(\%)$ & $4(3.3)$ & $14(15.9)$ & $36(22.7)$ \\
Limited-experienced, trained, $n(\%)$ & $64(53.3)$ & $24(27.2)$ & $29(18.3)$ \\
Limited-experience, non-trained, $n(\%)$ & $24(20)$ & $21(23.8)$ & $60(37.9)$ \\
& & & \\
IQR $=$ interquartile range & & & \\
\end{tabular}

Of the overall HCPs who identified $3(n=78)$ and 2 asynchronies ( $n=111)$, the percentage of trained HCPs was significantly higher than the non-trained group (3 asynchronies, 63 [81\%] trained vs 15 [19\%] non-trained, $P<.001 ; 2$ asynchronies, 72 [65\%] trained vs 39 [35\%] non-trained, $P=.034)$. Of the overall HCPs who identified 1 asynchrony $(n=116)$ and 0 asynchronies $(n=61)$, the percentage of non-trained HCPs was significantly higher (1 asynchrony, 55 [47\%] trained vs 61 [53\%] non-trained, $P=.02 ; 0$ asynchronies $=17[28 \%]$ trained vs $44[72 \%]$ non-trained, $P<.001$ ) (Fig. 2).

When we analyzed the trained versus non-trained groups separately, according to the number of asynchronies recognized correctly, the results showed that the percentage of HCPs who identified 3 asynchronies in the trained group was significantly higher than the percentage of HCPs in the non-trained group (63 HCPs [30.4\%] in the trained group vs 15 [9.4\%] in the non-trained group, $P<.001$ ). Similar results were obtained when we analyzed the percentage of HCPs who identified 2 asynchronies (72 HCPs [34.8\%] in the trained group vs 39 [24.5\%] in the nontrained group, $P=.034$ ) (Table 3 ).

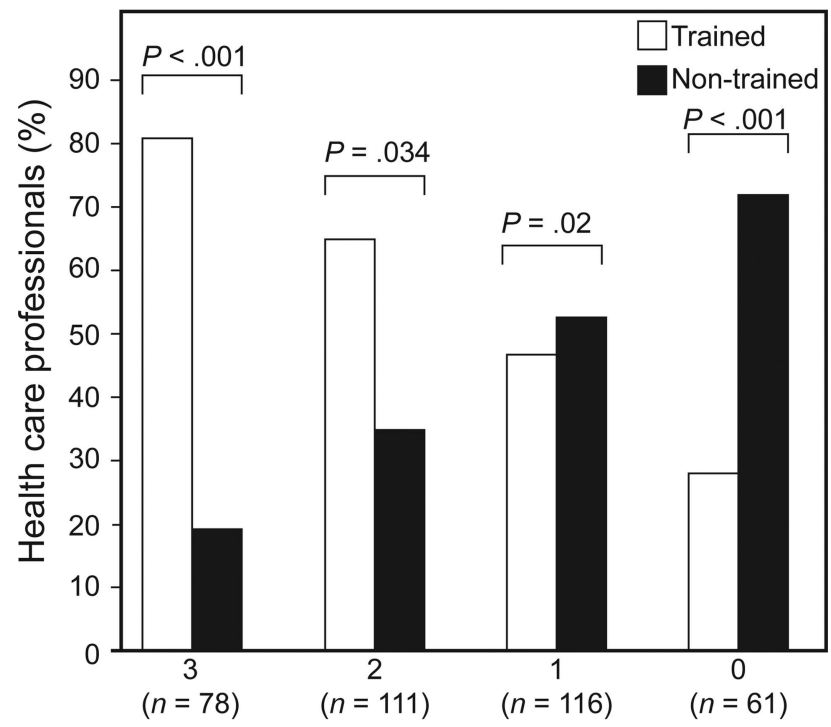

Number of correctly detected asynchronies

Fig. 2. Intra-group comparison between trained and non-trained health-care professionals according to the number of asynchronies recognized correctly.

Table 3. Comparisons of Trained Versus Non-Trained and Experienced Versus Limited-Experienced Health-Care Professionals According to the Number of PatientVentilator Asynchronies Recognized Correctly

\begin{tabular}{llccccc}
\hline \hline $\begin{array}{c}\text { Number of } \\
\text { PVAs Detected } \\
\text { Correctly }\end{array}$ & $\begin{array}{c}\text { Trained } \\
(n=207)\end{array}$ & $\begin{array}{c}\text { Non-Trained } \\
(n=159)\end{array}$ & $P$ & $\begin{array}{c}\text { Experienced } \\
(n=144)\end{array}$ & $\begin{array}{c}\text { Limited- } \\
\text { Experienced } \\
(n=22)\end{array}$ & $P$ \\
\hline 3 PVAs & $63(30.4)$ & $15(9.4)$ & $<.001$ & $32(22.2)$ & $46(20.7)$ & .73 \\
2 PVAs & $72(34.8)$ & $39(24.5)$ & .034 & $44(30.6)$ & $67(30.2)$ & .94 \\
1 PVA & $55(26.6)$ & $61(38.4)$ & .02 & $45(31.3)$ & $71(32)$ & .88 \\
0 PVA & $17(8.2)$ & $44(27.7)$ & $<.001$ & $23(15.9)$ & $38(17.1)$ & .77
\end{tabular}

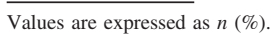

PVAs $=$ patient-ventilator asynchronies

Lack of recognition of any asynchrony was significantly higher in the non-trained group (17 HCPs [8.2\%] in the trained group vs 44 [27.7\%] in the non-trained group, $P<.001)$. This behavior was similar between the percentages of non-trained versus trained HCPs who identified only 1 asynchrony (Table 3). No statistically significant differences were found between groups when HCPs in the experienced and limited-experienced groups were compared according to the number of asynchronies identified correctly (Table 3 ).

A binary multiple logistic regression model was performed to determine whether prior training in mechanical ventilation was associated with the ability of HCPs to identify $\geq 2$ asynchronies. An odds ratio of 3.67 (95\% CI 1.93-6.96) was obtained, which means that trained HCPs who had prior training in mechanical ventilation increased, 


\section{IDENTIFying Ventilator Asynchrony Using WaVEFoRm ANALysis}

Table 4. Association of Training, Experience, and Profession With the Odds of Identifying $\geq 2$ Patient-Ventilator Asynchronies

\begin{tabular}{lccc}
\hline \hline \multicolumn{1}{c}{ Categories } & Odds Ratio & $P$ & $95 \%$ CI \\
\hline $\begin{array}{l}\text { Prior training in mechanical } \\
\quad \text { ventilation }\end{array}$ & 3.67 & $<.001$ & $1.93-6.96$ \\
Experience & 1.00 & .99 & $0.54-1.83$ \\
Physiotherapists & 1.73 & .15 & $0.81-3.71$ \\
Physicians & 1.53 & .25 & $0.73-3.20$ \\
Nurses & 1 & NA & NA
\end{tabular}

Nurses were considered the reference category.

$\mathrm{NA}=$ not applicable

nearly 4-fold, their odds of identifying $\geq 2$ asynchronies correctly. Neither experience nor profession was associated with increasing the odds of detecting asynchronies correctly. (Table 4).

The most recognized asynchrony by trained HCPs was double-triggering (73 of 92 trained physiotherapists [79.3\%] vs 11 of 28 non-trained [39.2\%], $P<.001 ; 32$ of 53 trained physicians [60.3\%] vs 11 of 35 non-trained [31.4\%], $P=.029 ; 40$ of 62 trained nurses [64.5\%] vs 46 of 96 non-trained [47.9\%], $P=.034)$. Although the percentage of trained HCPs who identified autotriggering and ineffective effort was higher than the percentage of nontrained for each profession, only within the physiotherapist group were the results statistically significant (Table 5).

\section{Discussion}

Considering that nearly $25 \%$ of patients receiving mechanical ventilation show some type of asynchrony during the time they spend receiving mechanical ventilation, ${ }^{7}$ maintaining an adequate patient-ventilator interaction is a key role that HCPs play in the ICU. ${ }^{16}$ However, learning how to identify and interpret asynchrony is not an easy task even for an experienced clinician. According to Younes et al, ${ }^{17}$ nearly $20 \%$ of asynchronies are unrecognized by clinicians, which is fairly similar to the results of this study (17\%). Our study also revealed that only a small percent- age of professionals (21\%) were able to correctly identify the 3 types of asynchrony shown in the evaluation videos.

Colombo et al $^{18}$ compared the ability between expert and non-expert ICU physicians to identify asynchrony by visual inspection of pressure versus time and flow versus time waveforms. The results showed that the rate of detection of asynchrony by waveform inspection was less than one third (28\%) in the expert group and $16 \%$ in the non-expert group when breath analysis of the pressure/ time and flow/time waveforms was performed, this difference being statistically significant. However, when the expert and non-expert groups were compared according to report analysis, no statistically significant differences were found $(P<.05)$. Their results ${ }^{18}$ as well as the report of Younes et $\mathrm{al}^{17}$ are consistent with our findings, both on the rate of recognition of asynchrony and on the association of ICU experience with identifying asynchrony, respectively.

The main strength of this study is the finding that HCPs who had prior training in mechanical ventilation increased their odds of identifying asynchrony correctly, which means that training in mechanical ventilation is a key factor associated with the ability of HCPs to identify asynchrony using waveform analysis. Also, to the best of our knowledge, this is the first multi-center study that has evaluated and compared the ability to identify different types of asynchrony by physicians, physiotherapists, and nurses. Another interesting finding is the fact that neither experience nor profession was shown to be associated with the ability of HCPs to identify asynchrony correctly using waveform analysis.

Our results also showed that although the correct answer for some of the videos (eg, double-triggering) could be easily inferred or guessed from the multiple-choice question, without specific training in mechanical ventilation, the number of trained HCPs who recognized this type of asynchrony was significantly higher than the number of those in the non-trained group.

Our study has several limitations. First, HCPs were asked to respond based on multiple-choice questions. Therefore, guessing should be a consideration for skewing the results in either direction. Second, we decided to simulate the

Table 5. Comparison Between Trained and Non-Trained Health-Care Professionals According to Profession and Type of Patient-Ventilator Asynchrony

\begin{tabular}{|c|c|c|c|c|c|c|c|c|c|}
\hline Asynchrony & $\begin{array}{c}\text { Trained } \\
\text { Physiotherapists } \\
(n=92)\end{array}$ & $\begin{array}{l}\text { Non-Trained } \\
\text { Physiotherapists } \\
(n=28)\end{array}$ & $P$ & $\begin{array}{c}\text { Trained } \\
\text { Physicians } \\
(n=53)\end{array}$ & $\begin{array}{l}\text { Non-Trained } \\
\text { Physicians } \\
(n=35)\end{array}$ & $P$ & $\begin{array}{l}\text { Trained } \\
\text { Nurses } \\
(n=62)\end{array}$ & $\begin{array}{c}\text { Non-Trained } \\
\text { Nurses } \\
(n=96)\end{array}$ & $P$ \\
\hline Double-triggering & $73(79.3)$ & $11(39.2)$ & $<.001$ & $32(60.3)$ & $11(31.4)$ & .03 & $40(64.5)$ & $46(47.9)$ & .034 \\
\hline Autotriggering & $62(67.3)$ & $6(21.4)$ & $<.001$ & $36(67.9)$ & $17(48.5)$ & .18 & $29(46.7)$ & $31(32.2)$ & .064 \\
\hline Ineffective effort & $66(71.7)$ & $14(50)$ & .041 & $26(49.0)$ & $15(42.8)$ & .83 & $24(38.7)$ & $33(34.3)$ & .50 \\
\hline
\end{tabular}

Values are expressed as $n(\%)$. 


\section{IdENTIFying Ventilator Asynchrony Using Waveform ANALysis}

videos to make sure that the asynchronies were based on the definitions described in the literature and to avoid the presence of artifacts in the waveforms caused by the presence of secretions, condensation, and cardiac oscillations. However, this is an aspect that must be considered as a limitation, because in a real clinical situation, HCPs must be able to distinguish asynchronies from these artifacts. Third, the perception of knowledge of HCPs in our country may not represent the reality of countries where the respiratory therapist is the primary $\mathrm{HCP}$ taking care of patients receiving mechanical ventilation. This aspect is important to mention because in our country the management, calibration, maintenance, and sometimes weaning of the mechanical ventilation is not performed exclusively by physiotherapists (also by nurses and physicians in some hospitals); this is due to the fact that respiratory therapy, as a career, does not exist in Chile and also because the competences required to manage mechanical ventilation have been acquired and developed by physiotherapists in the last decade. Fourth, we did not consider the level of training of each professional, which may vary depending on the educational institutions where he or she studied, and also so it will be interesting to know whether the results are similar to the results obtained from professionals in other countries.

\section{Conclusions}

HCPs who have specific training in mechanical ventilation increase their ability to identify asynchrony using waveform analysis. Neither experience nor profession proved to be relevant factors to identify asynchrony correctly using waveform analysis.

\section{ACKNOWLEDGMENTS}

We thank Jorge Olivares (Hospital San Jose, Santiago, Chile), Andrea Morales (Hospital de Talca, Talca, Chile), Jaime Figueroa, Christian Warnier (Hospital Militar, Santiago, Chile), Pilar Hermosilla (Hospital Clinico de la Florida, Santiago, Chile), Rodrigo Melo (Hospital de Chillán, Chillán, Chile), Carlos Nahuelqueo, Ines Mateu (Hospital de Coyhaique, Coyhaique, Chile), Pablo Pinto (Hospital San Juan de Dios, Curico, Chile), Gonzalo Varas (Hospital San Martin de Quillota, Quillota, Chile), Sharon Arias (Hospital Clínico de Magallanes, Magallanes, Chile), Andres Rey (Hospital de Arica, Arica, Chile), Marcela Schulz (Hospital de Temuco, Temuco, Chile), Patrick Sepulveda, Eduardo Gonzalez (Hospital San Juan de Dios La Serena, La Serena, Chile) for helping with the assessment and data gathering process. We also thank Ro- drigo Cornejo MD (Hospital Clinico Universidad de Chile, Santiago, Chile) for advice on the manuscript and Michelle Cude and Piry Salgado for helping in the translation of the manuscript.

\section{REFERENCES}

1. Kondili E, Prinianakis G, Georgopoulos D. Patient-ventilator interaction. Br J Anaesth 2003;91(1):106-119.

2. Epstein SK. How often does patient-ventilator asynchrony occur and what are the consequences? Respir Care 2011;56(1):25-38.

3. Yang LY, Huang YC, Macintyre NR. Patient-ventilator synchrony during pressure-targeted versus flow-targeted small tidal volume assisted ventilation. J Crit Care 2007;22(3):252-257.

4. Chao DC, Scheinhorn DJ, Stearn-Hassenpflug M. Patient-ventilator trigger asynchrony in prolonged mechanical ventilation. Chest 1997; 112(6):1592-1599.

5. Imanaka H, Nishimura M, Takeuchi M, Kimball WR, Yahagi N, Kumon K. Auto-triggering caused by cardiogenic oscillation during flow-triggered mechanical ventilation. Crit Care Med 2000;28(2): 402-407.

6. de Wit M, Miller KB, Green DA, Ostman HE, Gennings C, Epstein SK. Ineffective triggering predicts increased duration of mechanical ventilation. Crit Care Med 2009;37(10):2740-2745.

7. Thille AW, Rodriguez P, Cabello B, Lellouche F, Brochard L. Patient-ventilator asynchrony during assisted mechanical ventilation. Intensive Care Med 2006;32(10):1515-1522.

8. Arellano D. Ventilación mecánica: generalidades y modalidades tradicionales. Kinesiología 2006;25(4):17-25.

9. Georgopoulos D, Prinianakis G, Kondili E. Bedside waveforms interpretation as a tool to identify patient ventilator asynchronies. Intensive Care Med 2006;32(1):34-47.

10. Nilsestuen JO, Hargett KD. Using ventilator graphics to identify patient-ventilator asynchrony. Respir Care 2005;50(2):202-234; discussion 232-234.

11. de Wit M. Monitoring of patient-ventilator interaction at the bedside. Respir Care 2011;56(1):61-72.

12. Carlucci A, Pisani L, Ceriana P, Malovini A, Nava S. Patient-ventilator asynchronies: may the respiratory mechanics play a role? Crit Care 2013;17(2):R54.

13. Sinderby C, Liu S, Colombo D, Camarotta G, Slutsky AS, Navalesi P, Beck J. An automated and standardized neural index to quantify patient-ventilator interaction. Crit Care 2013;17(5):R239.

14. Branson RD, Blakeman TC, Robinson BR. Asynchrony and dyspnea. Respir Care 2013;58(6):973-989.

15. Chatburn RL. Classification of ventilator modes: update and proposal for implementation. Respir Care 2007;52(3):301-323.

16. Sassoon CSh. Triggering of the ventilator in patient-ventilator interactions. Respir Care 2011;56(1):39-51.

17. Younes M, Brochard L, Grasso S, Kun J, Mancebo J, Ranieri M, et al. A method for monitoring and improving patient: ventilator interaction. Intensive Care Med 2007;33(8):1337-1346.

18. Colombo D, Cammarota G, Alemani M, Carenzo L, Barra FL, Vaschetto R, et al. Efficacy of ventilator waveforms observation in detecting patient-ventilator asynchrony. Crit Care Med 2011;39(11):2452-2457.

This article is approved for Continuing Respiratory Care Education credit. For information and to obtain your CRCE

(free to AARC members) visit www.rcjournal.com

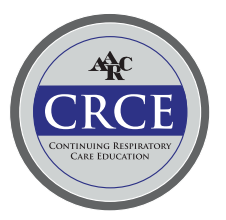

